\title{
Industrial designers' thinking in the stage of concept generation for social design: themes, strategies and modes
}

\author{
Yiqi Xiao ${ }^{1}$ (D) . Chenhan Jiang ${ }^{2}$
}

Accepted: 13 January 2022 / Published online: 10 February 2022

(c) The Author(s), under exclusive licence to Springer Nature B.V. 2022

\begin{abstract}
Social design produces strategic, systematic solutions or new cultures as a response to the complexity of social changes and, in consequence, designers who are used to the traditional way of ideation may not be well prepared for the requirements of social design. This paper identified the characteristics of the concept generation of industrial design students participating in a social design practice as novices. Using the think-aloud protocol, we collected the conversations and self-reports of students $(n=42)$. We then conducted a qualitative analysis of the designers' activities with the inductive and deductive coding method. An effect of prior knowledge was found on the themes of concept, as well as on the concept generation strategies and modes that industrial designers would prefer. Through a factor analysis of the frequency of students' design activities, six concept generation strategies were clustered. There were eight concept generation modes for social design we summarized with the journeys of designers' activities. This study also revealed the effect of concept generation strategies and modes of industrial design students on the quality of their social design concepts. The results may also shed light on the question how we can foster the quality of industrial designers to adapt to the extension of disciplinary boundary in design.
\end{abstract}

Keywords Concept generation · Social design · Industrial designer $\cdot$ Novice $\cdot$ Design ideation

Yiqi Xiao and Chenhan Jiang contributed equally to this work.

Yiqi Xiao

vincentxiaoyiqi@163.com

Chenhan Jiang

chenhan0713@126.com

1 College of Communication and Art Design, University of Shanghai for Science and Technology, No. 516, Jungong Road, Shanghai 200093, China

2 College of Architecture and Urban Planning, Tongji University, Shanghai, China 


\section{Introduction}

Social design, as a term, is becoming popular, as many problems of our society arise along with the development of industrial civilization and post-modern consumerism (Whiteley, 1994). It aims at a shift of the topics in design research from serving the needs of consumers to the sustainability of social relationships (Manzini, 2015) and also to the responsible design for globally marginalized communities (Papanek, 1985). As a result, the design outputs, working methods and the design thinking required for social design are different from those for traditional industrial design (ID). Currently, a number of social design education programmes in universities has been carried out. Some of them are modified from projector studio-based learning environments of design schools (Easterday et al., 2018). Industrial designers can play an important part in such programmes or courses.

Conventionally, industrial design is conducted to create the commodities and services that are attractive enough to activate consumer behaviors and the consequent extended reproduction. This is an objective for which designers should make the material objects look good and appealing in some cultural contexts. In addition to this, good industrial designs should evoke a variety of consumer experiences (Mozdzynski, 2014), and thus the idea of user-centered design-which calls for the empathy with real needs of humanbecomes prevailing as it appears to be an effective method to enhance such experiences. These two objectives are what the industrial design methodologies in terms of research and thinking are primarily developed for. The integrated system of knowledge for ID students is therefore traditionally comprised of the hand-on experiences in market opportunity identification, product structure, manufacturing, prototyping and usability test. However, according to the visions of social design presented by Margolin (2002), designers are encouraged to seek the solution for a wider scope of problems in human society with both tangible and intangible design initiatives, and work for the social well-being. Here, it is obvious that a continuum of design objectives from market-oriented to social-oriented exists. Designers should adapt to this new role to cope with the more complex social and societal problems (Bobbe et al., 2017).

The main body of this paper describes a case study of teaching by which we investigated how the industrial designers, in particular the ID students, generate design concepts for social design. Our study on concept generation is comprised of three aspects: students' preferences for theme, the strategies and the modes of idea generation. For the instructors, these work is made to give insight into the effects of particular design expertise on the learning of social design. With the identification of the thinking characteristics of ID students, suitable educational measures can be easier to find to facilitate the leap in the professional ability of novice designers.

\section{Literature review}

\section{Social design: objectives and works}

The clear-cut boundary, form and limits of social design have been discussed for a long time though, its definition still remains amorphous and controversial. Some design practices working for socially responsible and responsive design have explicitly expanded design "beyond its traditional core and scope" (Chen et al., 2016). Following the idea of Papanek (1985), a socially responsible design is usually practical and problem-based. It 
advocates the design for sustainable and localized environment or economy in order to benefit the local communities (Melles et al., 2011). On this account, it should be viewed as a kind of social innovation considering that it can meet social needs, safeguard the common good or guide the technological innovation (Jégou \& Manzini, 2008). For example, we can design a product that can coerce, persuade, seduce or decide for somebody to change their bad behavior instead of reinforcing it, thereby having a positive social impact (Tromp et al., 2011).

The value of socially responsive design is addressed in the co-design process in which designer and local citizens are involved. Being different from socially responsible design, it suggests the role change of social designers from the facilitator of solutions that meet local needs to the catalyst for creating socially responsive design initiatives in collaboration with experts and local users (Thorpe \& Gamman, 2011). This claim exhibits a sense of design activism (Armstrong et al., 2014). It describes design as a progress of communicating and prototyping but not as a result.

In fact, the working method of social design in a narrow sense must be only action research, through which a final solution can, but is not necessarily required to be developed. According to Markussen (2017), the value of social design lies in improving life conditions or making issues visible, by means of designing for the community, identity and interpersonal relationship. It is characterized by small-scale design outputs and microlevel transformations in lifestyle (Koskinen \& Hush, 2016), aiming at the improvement of mental well-being. Design cases in our study include both social innovation and the "real" social design.

The social design topics (Akdur \& Kayga, 2019) include but are not limited to: (1) working for small community, especially the rural area (Wang et al., 2016) or local community (Lange et al., 2014); valuing the use of local intelligence or seeking "low budget and hence low technology solutions supported by local creativity and innovation" (Szentpéteri, 2009); (2) vulnerable groups like the homeless people (Melles \& Howard, 2012) and confined social group; (3) global issues (Philips et al., 2016). Designers are encouraged to work together with multi-faceted stakeholders (DiSalvo et al., 2011) such as social innovation platforms (Yang \& Sung, 2016), local partners (Gaudio et al., 2016), or the government, industry organizations, and even civil society organizations (Carney et al., 2012). In order to "support the contextual understanding of the communities that participate in research" (Miettinen \& Sarantou, 2017, p. 7), designers are recommended to adopt ethnographic methods such as participant observation, group interviews, photograph and video documentation (Miettinen \& Sarantou, 2017). Note that these methods should be situated, or more precisely, be capable of "altering in accordance with the situation at hand" (Kang, 2016, p. 72). Also, personal methods are sometimes useful. Tromp (2013) introduced the social implication design method for design proposals by analyzing a phenomenon and the product influence.

\section{The study on design concept generation}

How does the intermediate stage between problem input and design output work? This is an open question because that stage is always nebulous, driven by designer's experiences. These experiences are part of designers' implicit knowledge and thus very hard to articulate. The theory of reflective practitioner separates a design activity into four fundamental steps: naming, framing, moves and evaluating (Dorst, 1997). The first two steps are critical to a successful concept generation. As we know, many of the problems under social 
circumstances are ill-defined (Cross, 2010), open, complicated, dynamic and networked (Leeuwen et al., 2020). To reframe, divide and transform these problems, a practitioner must first identify the contextual factors, select them in a certain way and then define a design issue. In practice, there is a continuous interaction effect between naming and framing, contributing to the translation of a great deal of contextual information into an easy-tounderstand problem scenario (Schön, 1983).

The process of concept generation, if presented as a diagram, is the one which proceeds from divergence to convergence (Fig. 1). A question is therefore raised that how these two kinds of action generally interact with each other in concept generation. Although a number of descriptive models for this question (Banath, 1996; Cross, 2008; Pugh, 1991; Liu \& Chakrabarti, 2003) are seen, the unified model has yet to be found. In fact, as the description of concept generation, reflection and evaluation activities, divergence and convergence will be performed differently between design teams and in different design stages. Researchers have also summarized the approaches and toolkits (Cross, 2010; Dorst \& Valkenburg, 1998; Finke et al., 1992) for design ideation, as well as the blocks and tackles (Shah et al., 2000) in the ideation process. These are utilized by designers in any of the stages — naming, action and reflection (Schön, 1983) — to facilitate design ideation. Due to the differences in the teams, design requirements and thinking activities, the whole journey of a concept generation including many divergence-convergence processes is diverse. Different working styles can be abstracted from the diversity of journey (Kim \& Kim, 2015).

In addition to the research on the concept generation process, researchers also pay attention to designer's strategies in seeking the solutions for a design problem. Such a strategy is characterized by the difference in the frequency of some design activities (Kruger \& Cross, 2006), which reflects what approaches the designer use more to think and decide. Kruger and Cross (2006) concluded four strategies: "problem driven", "information driven", "solution driven" and "knowledge driven". Problem driven strategy focuses on both data gathering and identifying constraints. Information driven strategy puts emphasis on data gathering. Solution driven strategy focuses on generating and assembling solutions; and knowledge driven strategy utilizes prior personal knowledge.

Another research domain about concept generation is the differences in seeking solutions between novice and expert designers. Regarding the problem framing, expert designers are often better at clarifying a problem (Dwarakanath \& Wallace, 1995) by collecting related information, giving it a meaning (Bryson, 1991) and adding the constraints (Atman $\&$ Bursic, 1998), and of course they work for these with longer time (Atman, 1999). In terms of the design activity, novices prefer the pattern of trial-and-error for design iteration (Atman et al., 2005). Not only do they alternate between activities less frequently (Ahmed et al., 2005), but are also not so active to use design heuristics (Yilmaz \& Seifert, 2011) and start mutual triggering (Hu et al., 2015). Expert designers were found to use conversation activities in relation to cognitive process more effectively than novices (Kiernan et al., 2020). We are interested in the thinking of ID students who have received technology education and thus are of prior knowledge in engineering and product design. Due to the lack

Fig. 1 The diagram of divergence-convergence process

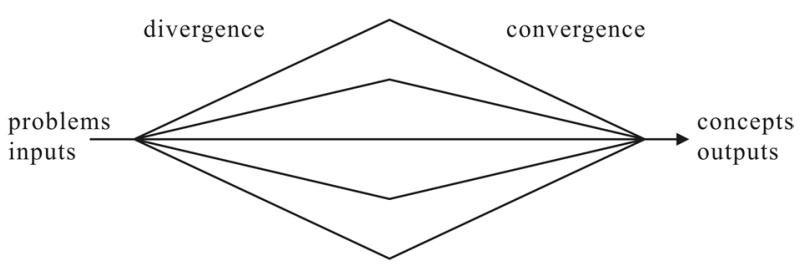


of experience in social design projects, however, ID students may distinguish themselves concerning design ideation from the expert designers.

\section{Research design}

\section{Research objectives and questions}

The objective of this research is to characterize ID students' thinking of concept generation when engaged in social design projects and to explore the impacts of the characteristics of ID students' thinking on the quality of generated concepts. This research can have some implications for design thinking in college education under the goal of integrated innovation.

As the features of concept generation, strategies and modes were important topics in previous studies (Kim \& Kim, 2015; Kruger \& Cross, 2006). We identified a number of divergent and convergent activities and investigated the concept generation strategies preferred by ID students through the frequency of these activities. By analyzing how these activities construct the journey of concept generation, we also can find common characters whereby some generation modes can be extracted. In addition, we intend to observe the characteristics of design themes proposed by ID students engaged in social design for the first time. The above three are the main research questions of this study.

\section{Participants}

The research data of this study were collected from undergraduate teaching cases. We used convenience sampling to recruit students who chose the "Interaction Design" course. The students are of the same grade and major. In addition, the grade points of the students are evenly distributed in the range from 2 to 4, which can reduce the sampling variability. Forty-two $(n=42)$ third-year students (16 males, 26 females) majoring in ID participated in our study. The participating students were asked to work in pairs, so there were 21 teams. We used $\mathrm{T} 1 \ldots \mathrm{T} 2 \ldots$ to represent the teams and $\mathrm{P} 1 \ldots \mathrm{P} 2 \ldots$ to represent the participating students. The undergraduate training program limited students' interest in product innovation design and product engineering, with the course content oriented toward controlling production costs, identifying market opportunities, managing the supply chain and designing user experiences. None of the participants had previously engaged in social design or even social innovation projects.

\section{Design workshop}

We ran the course from May 2020, when COVID-19 had already spread worldwide. Continuous news reports enabled students to recognize the situation, and they might have gained a direct understanding of the social problems from what they underwent. We assigned a subject called "Post-epidemic Social Design", allowing students to freely select a social issue as the theme and discuss it without teacher involvement. In view of the particularity of social design, we specifically made the design requirements distinguishable from those of industrial design (see Table 1).

The course lasted for six weeks, during which a 4-week design workshop was set to generate design proposals. Team members met with each other on Monday every week 


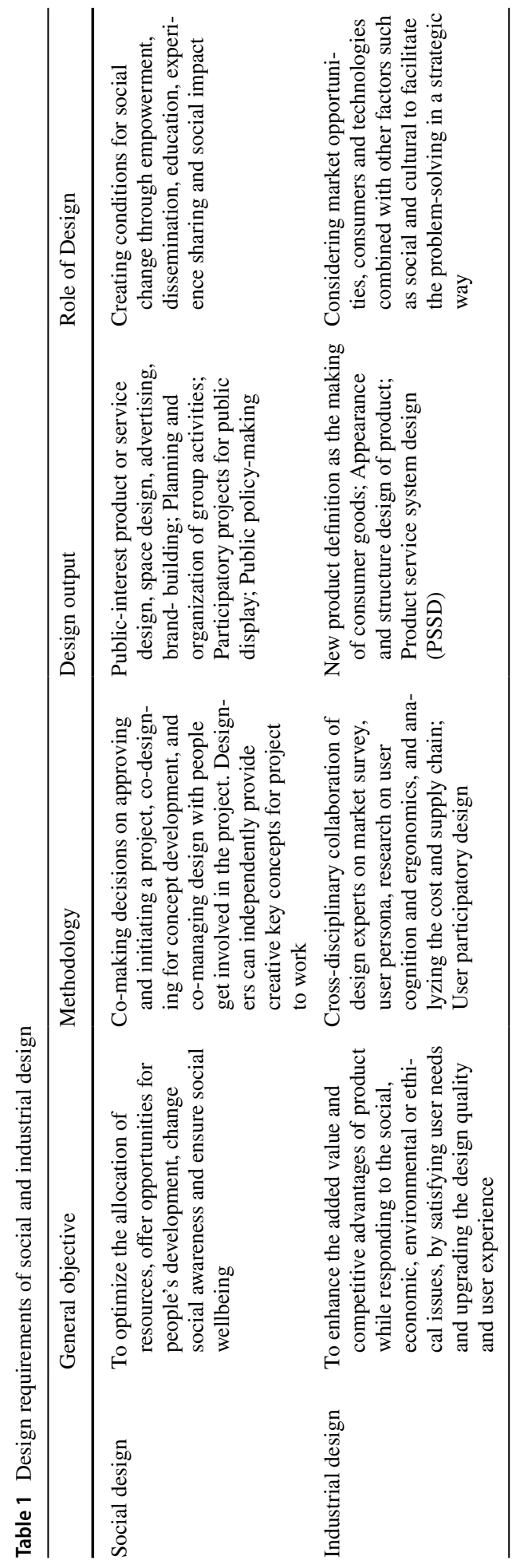


for an online discussion, in line with public health measures. After that, they had to conduct the field study once a week. Field study is indispensable to a social design project, as it is essential for designers to define the problem scenario, understand stakeholders and thus reframe the design issue. Without contact and collaboration with locals, students would have no opportunity to experience the process of solving authentic social problems. Through contextual interviews and codesign, they were each provided with ethnographic data, participatory design proposals and design suggestions. In the online discussion, team members shared the data individually collected before generalizing, evaluating and debating on it, which acted as the basis of concept generation. This can be seen as a full practice of design and reflection. To ensure the same time reserved for concept generation across teams, team members were forbidden to talk about the design process or exchange data other than during in-class discussion. We used the think-aloud protocol to collect data from the teams' discussions. The time limit for each team's discussion was $20 \mathrm{~min}$, so the recording duration in total was $20 \mathrm{~min} \times 4$ weeks $\times 21$ teams $=1680 \mathrm{~min}$. Participating students were allowed to draw sketches to assist in thinking and concept expression, but these sketches were not collected as data, only as a tool to show the concepts more clearly to researchers.

\section{Self-statement}

After the design workshop, a self-statement session was set up to assist researchers in abstracting more characteristics of the ideation process. For this purpose, an open questionnaire was prepared to guide participants to concisely state their design concept with respect to the social problem, main content, target users and social value, and to briefly report the thinking process with a review of the design motivation, difficulties, deficiencies and reference materials. The self-reflection session lasted for 10-15 $\mathrm{min}$ for each team. The questionnaire is given in Appendix. The statements of students were used as supplementary materials for encoding the design themes and understanding the concept generation modes.

\section{Coding methods}

We used protocol analysis to encode the verbatim transcripts of the in-class discussions. The main part of the coding scheme of this study is inductive, aiming at defining the categories of design themes and the elements of designers' cognitive activities that will be the basis for analyzing the strategies and modes of idea generation in social design. A theorydriven coding category first evolved, then the definition of each category was refined, or confirmed by deductive coding. Thus the entire coding scheme was finally developed.

The coding scheme we utilized to classify the design themes of social design was developed based on previous studies. A framework formulated with Markussen's definition of social design (2017) describes a social design initiative from five perspectives: aim, social value, modus operandi, locus of innovation and social effect. The first two perspectives are more direct in explaining the theme of a social design concept. In our study, a new perspective defined as "final deliverable", including industrial product, interaction design, service design and public activity, was added to the coding scheme. For the aim of social design, a taxonomy reported in Akdur and Kaygan's work (2019) elaborated several objectives of social design including develop solutions, organize, make visible and empower. We adopted this taxonomy and the description of each category in our research. We used open coding to interpret related utterances, extracting 
the sentences related to describing social values. Axial coding was then performed to merge the coding items and reduce them into seven subcategories, as shown in Table 2.

The coding scheme of the designer's activities was drafted based on previous studies related to reflective practitioners (Dorst, 1997) and divergence-convergence paradigms (Cross, 2008). Similar to the process of designing physical objects, a social design process consists of the analysis of social issues, idea generation and the execution of clearly described plans or measures that benefit people or the whole society. There are particular design activities for these three main stages. Through a primary encoding with the transcripts of three teams, we identified three main activities, problem definition, concept construction and design expression, each consisting of a divergent (i.e., give a proposition) and a convergent activity (i.e., judgment on the proposition). Moreover, we not only observed many propositions from the transcript but also noticed some judgments on the feasibility and necessity of the propositions in every stage of the design process. In this regard, we added "value proposition" (Levina \& Ross, 2003) to the coding system. Table 3 provides a brief explanation and an example of each coding category. This study interprets the differences in concept generation strategies by computing the frequency of the coding categories.

To summarize the concept generation modes of ID students, we employed cognitive maps (Kim \& Kim, 2015; Roy et al., 2012) as the method to abstract the journey of a concept generation process. A cognitive map is a description method that "provides a view of the entire structure of the cognitive elements in a design process" (Kim \& Kim, 2015 , p.14). It can be visualized as a network with nodes that are connected by links of relations that are used to represent the dynamic process of cognitive and thinking activities. According to the coding system shown in Table 3, we structured the journey of each team's concept generation process with the encoded activities, and as such, we could closely monitor how an idea evolved. An example of such a journey is depicted in Fig. 2, where each encoded item is expressed as a different type of figure in accordance with its coding category. We used squares, diamonds, circles and triangles to represent the problem definition (PD), concept construction (CC), design expression (DE) and value proposition (VP). Different from the standard cognitive map, the journey map focuses on the sequence of activities rather than their relationship network. In Fig. 2, each column of figures can be viewed as a timeline; the location of a figure is the point of time when the activity occurred during discussion period. An activity in each column is related only to the activities before and after its occurrence. The unrelated, suddenly emerging activities are placed in another column, according to the time point of their occurrence. The straight line penetrating many figures denotes the sequence of activities that leads to a final design proposal.

To derive the generation modes, we marked the milestones in each concept generation process. Milestones are visually represented as nodes. It can be a well-defined problem, a core concept, the conclusive judgment on a proposition, or even the acquisition of important external knowledge. A milestone should play a decisive role in producing the final concept. The discussions that did not lead to the establishment of the final concept and those not further developed were consequently not regarded as milestones. Figure 3 illustrates the ideation process of one team by highlighting the milestones in its work. We classified the generation processes with similar journey structures formed by milestones into one category, using the analytical inductive method. Thus, some concept generation modes were obtained and named according to the characteristics of their structure. 


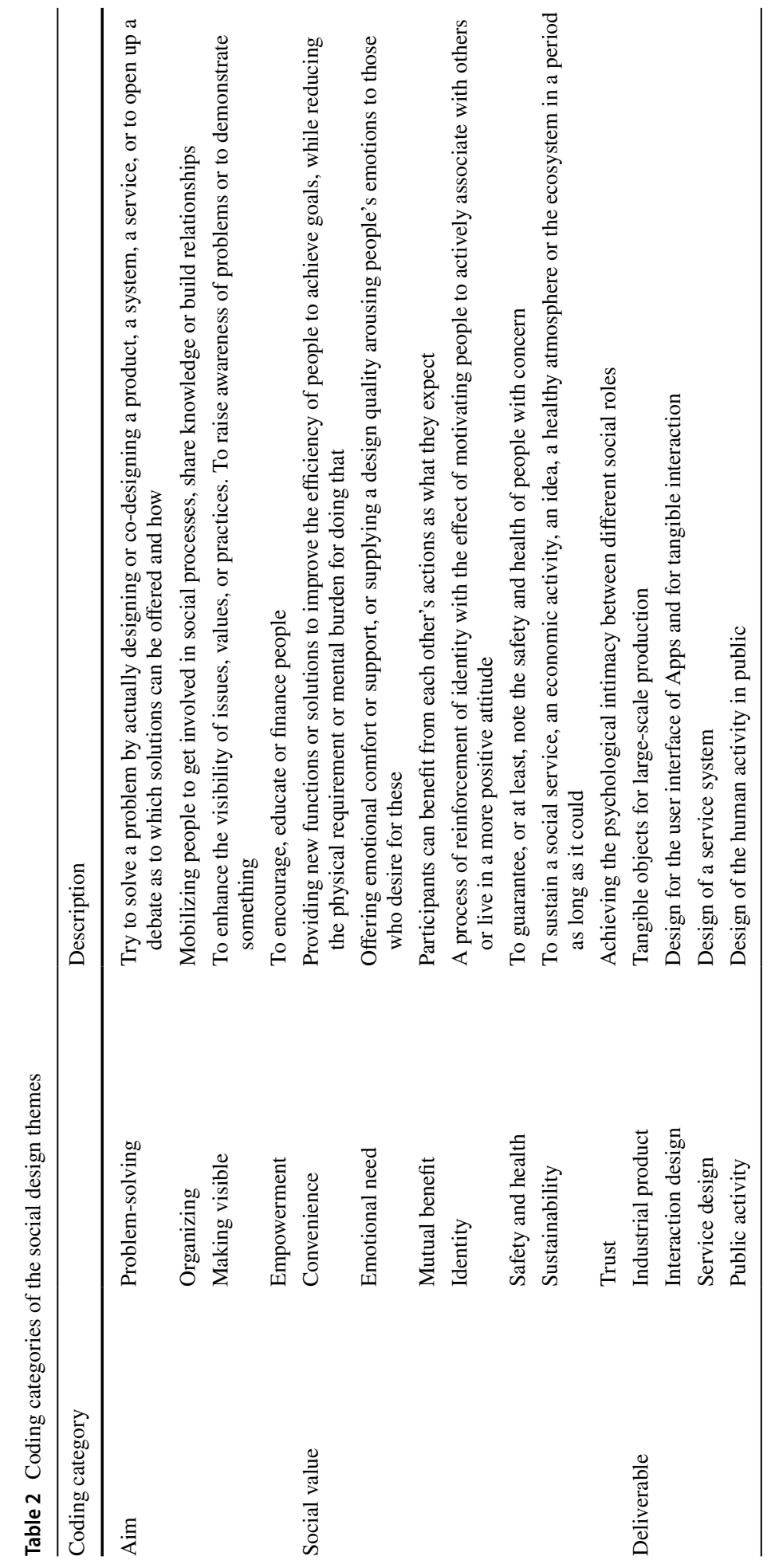




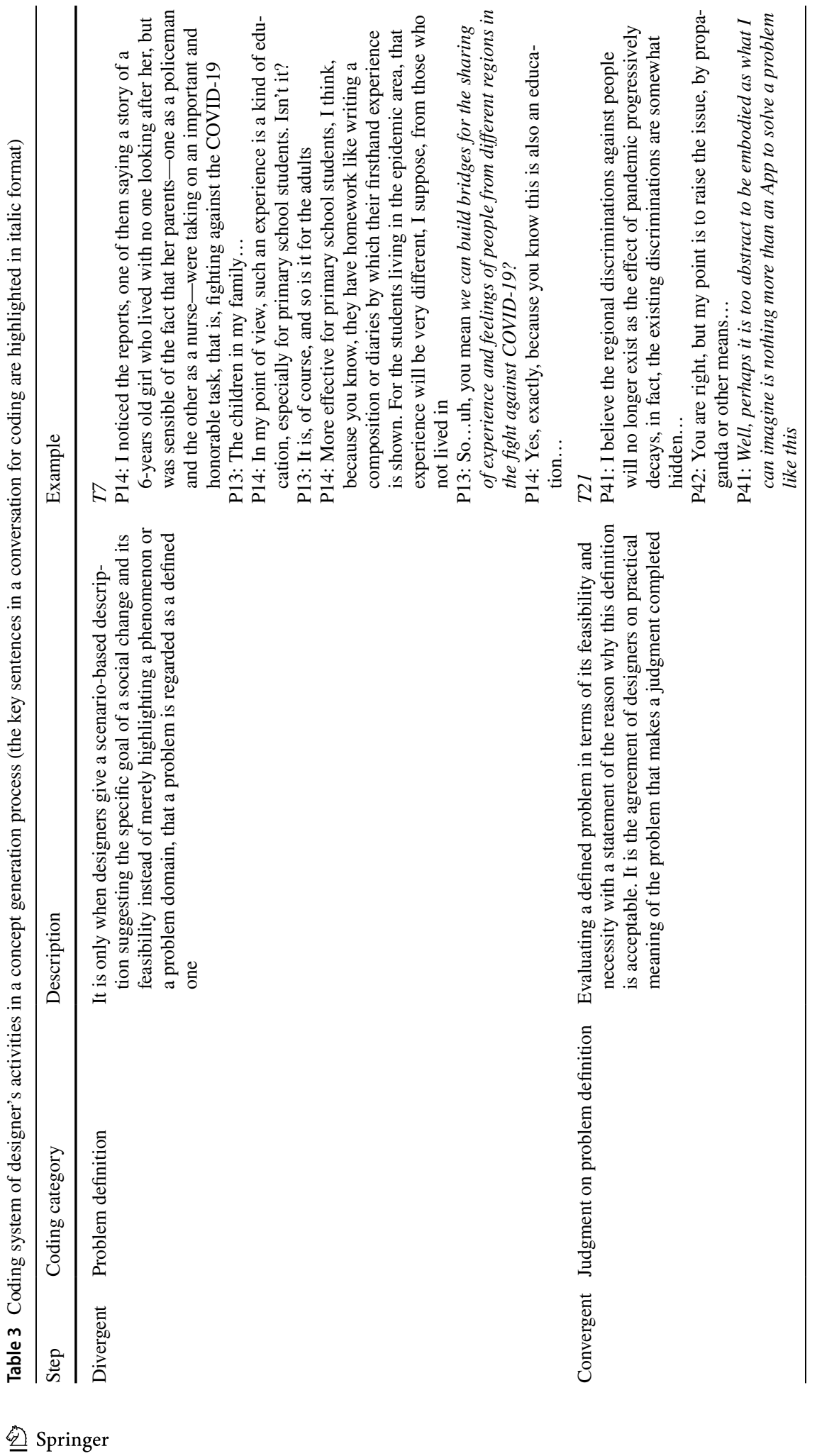




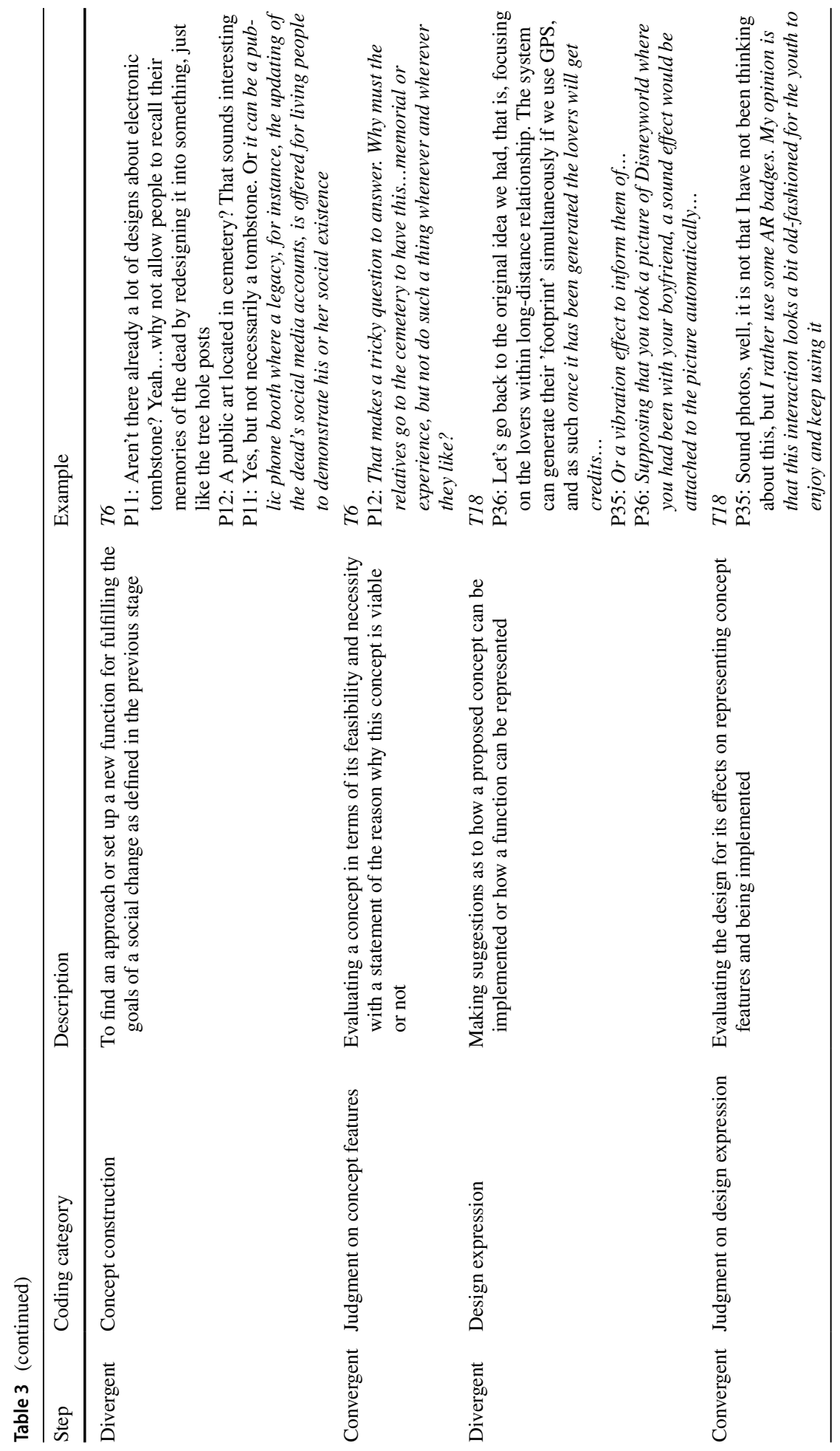




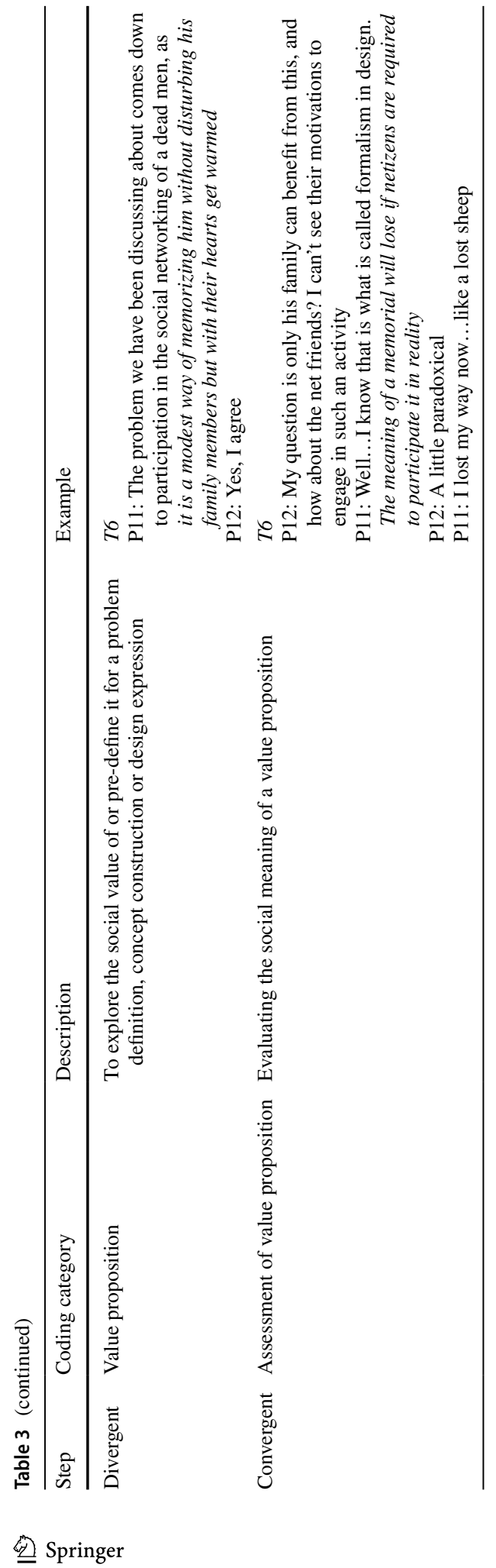




\begin{tabular}{|c|c|c|c|c|c|c|c|}
\hline$\square$ & $n$ & $\diamond$ & 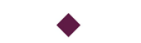 & 0 & 0 & $\triangle$ & $\Delta$ \\
\hline $\begin{array}{l}\text { Problem } \\
\text { definition }\end{array}$ & $\begin{array}{c}\text { Judgment on } \\
\text { definition }\end{array}$ & $\begin{array}{c}\text { Concept } \\
\text { construction }\end{array}$ & $\begin{array}{c}\text { Judgment on } \\
\text { concept } \\
\text { features }\end{array}$ & $\begin{array}{c}\text { Design } \\
\text { expression }\end{array}$ & $\begin{array}{c}\text { Judgment on } \\
\text { expression }\end{array}$ & $\begin{array}{c}\text { Value } \\
\text { proposition }\end{array}$ & $\begin{array}{l}\text { Assessment } \\
\text { of value } \\
\text { proposition }\end{array}$ \\
\hline
\end{tabular}

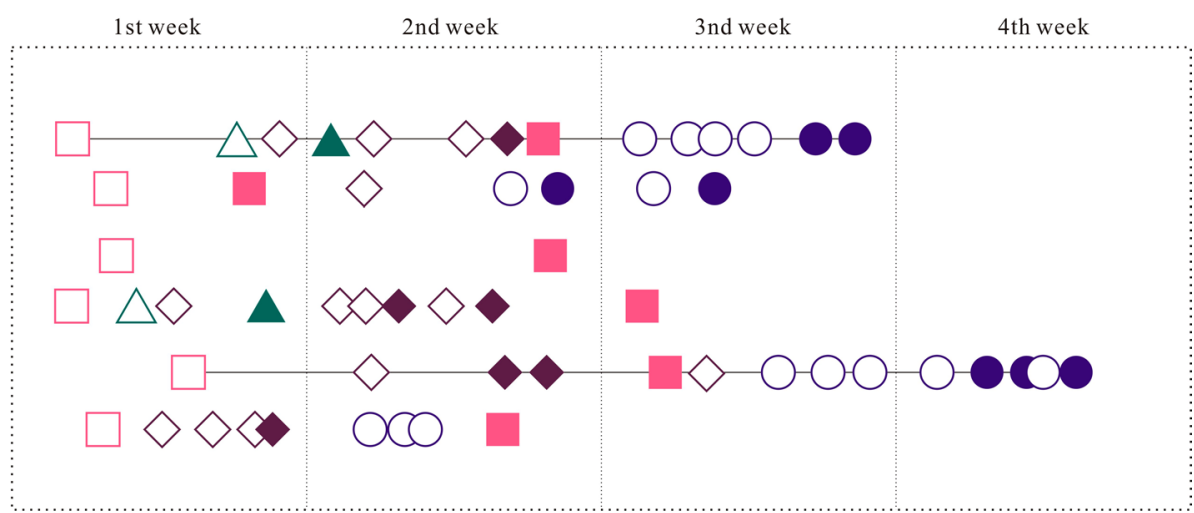

Fig. 2 Partial journey map of T1

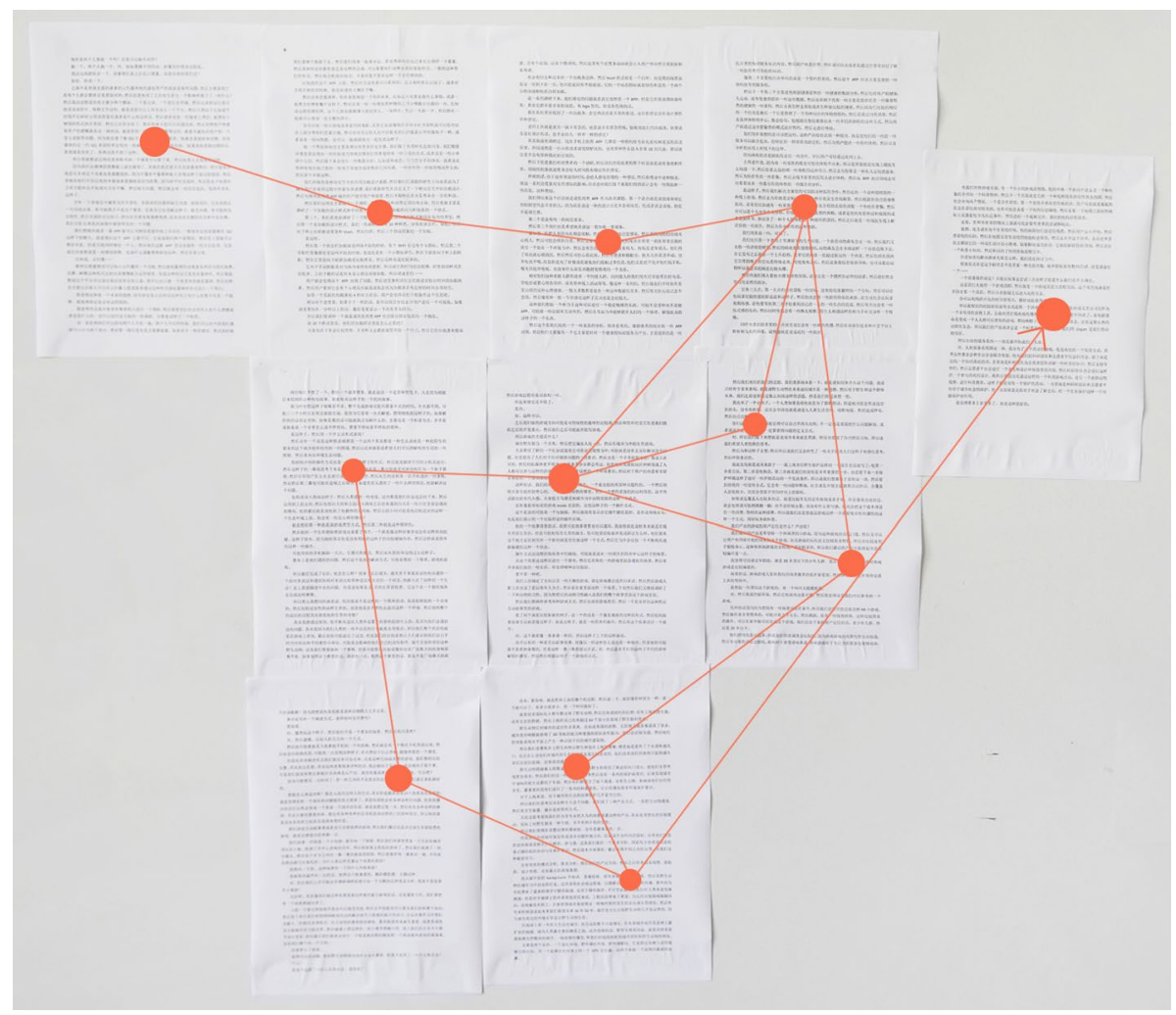

Fig. 3 Ideation process of Team 10, where the milestones reveal important steps of concept generation that directly contribute to the final concept 


\section{Data analysis}

The two researchers served as interpreters engaging in the protocol analysis. All the think-aloud data were processed using Atlas.ti software (version 8.2.30). At the beginning, the two researchers cooperated on the primary coding of design activities. Then, they each encoded the protocols of the 21 teams twice. There was a time gap of one week between the first encoding and the second encoding. This setting could help enhance the reliability of encodings and provide an opportunity for coders to verify the definition of each category. The two encoded protocols were then compared, and the level of agreement was calculated with Cohen's kappa coefficients. The $\kappa$ value throughout all coding categories was above 0.80 , indicating substantial agreement of the coding results. The milestones also were encoded in this manner. Considering that the encoded results reached an acceptable level of interrater agreement, the two researchers organized a meeting to arbitrate and adjust the disagreements until the final protocol was obtained.

With the encoded results on design activity, the frequency of each category of activity was processed with the factor analysis method. The common factors were first extracted, and a factor model was established to calculate the common factor scores. In the next step, cluster analysis on factor scores was conducted to reveal the different concept generation strategies. Additionally, we compared the difference between divergent and convergent activity in frequency of occurrence by performing the Wilcoxon signed-rank test. By doing so, we can understand which categories of activity were more preferred by ID students when generating social design concepts.

To examine the influence of the characteristics of ID students' thinking on the generation process and the results of concepts for social design, twelve experts with at least three years of experience in the social design field were invited to assess the quality of the students' concepts. Consistent with prior research on evaluating designers' performance (Luo $\&$ Dong, 2017), the quality of concepts was measured based on two dimensions: originality and practicality. Originality refers to the degree of innovation and attractiveness of a concept, while practicality refers to how practical a concept is in responding to social problems. Experts were provided with sketches of concepts and students' self-statement questionnaires. With these materials, they rated the concepts using a 10-point scale, with 1 indicating "not original/practical at all" and 10 indicating "extremely original/practical". To reveal the potential effects of concept generation strategies and modes on expert scores, we performed Mann-Whitney's U test and the Kruskal-Wallis H test to calculate the differences in expert scores between different strategies and modes.

\section{Study ethics}

Ethical approval and permissions were obtained from the university's academic ethics and discipline supervision committee. The objectives and methods of the study were provided to the committee members and the dean of the industrial design department in advance. All data collection processes were completed under their supervision to ensure that the students were not forced, and there were no behaviors of linking the study results with the course grade. Students signed a letter of concern to participate in the research, and they were told explicitly that they could quit the study at any time without explanation. During the research, the names of the participating students were not revealed to protect their 
identities. In addition, the webcam embedded in the student's laptop was required to be turned off during online discussion.

\section{Findings}

\section{Themes}

Our coding scheme for design themes is shown in Table 2. The coding categories for the aim of concepts were derived from the taxonomy presented by Andur and Kaygan (2019). With more than one aim coded for many of the concepts, it was difficult to demarcate the 21 design concepts and group them into independent categories of aim. We therefore examined the frequency of each aim. It was found that there were more concepts proposed with the aim of problem solving $(\mathrm{n}=16)$ than with organizing $(\mathrm{n}=10)$, making visible $(\mathrm{n}=9)$ or empowerment $(\mathrm{n}=6)$ (see Fig. 4). Among the 16 concepts aimed at problem solving, 15 attempted to make a functional innovation to respond to the problem definition, except the one submitted by T6.

Participating students preferred to propose concepts with the social values of convenience, safety and health, and satisfaction of emotional needs. This result is supported by the frequency with which students' concepts are encoded as these social values. More details can be seen in Fig. 4. With respect to the final deliverables, ID students showed a preference for interaction design and service design. We observed 15 concepts containing interaction designs, 11 belonging to service design, 8 involving public activity, and 8 focusing on or making use of industrial products. T18's concept was a typical interaction design, while T5, T12 and T20's work was related only to service design. T3 and T15 resorted to product design to respond to social concerns. Many of the concepts were encoded by more than one category of deliverables and social values. In general, the ID students tended to propose a "tangible" design to eliminate problems and change reality. They would consciously design "a usable thing" as the supplement to a service system or the aid to the planning of a group activity.

Fig. 4 The frequency graph of the coding categories about theme

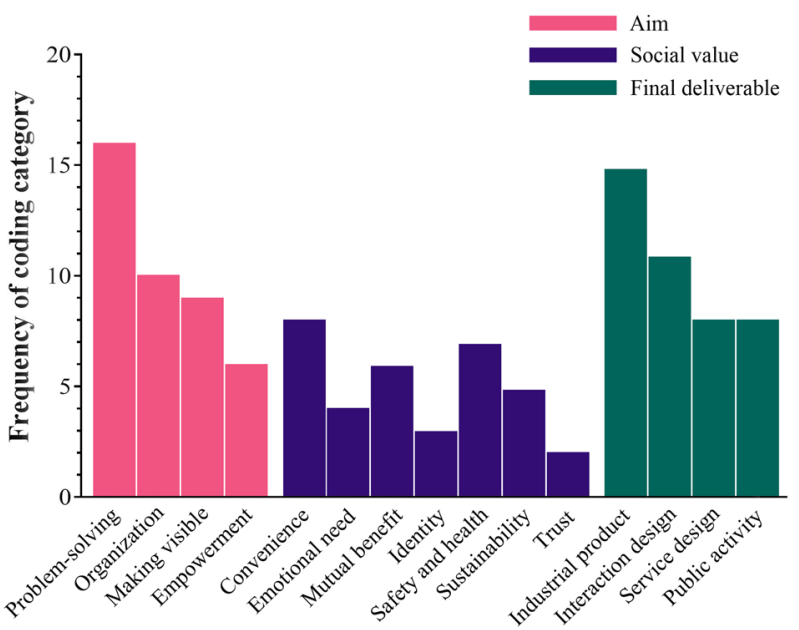


Table 4 Communalities of the coding categories of designers' activity

\begin{tabular}{lll}
\hline Activity category & Initial & Extraction \\
\hline Problem definition & 1.000 & 0.727 \\
Judgment on problem definition & 1.000 & 0.630 \\
Concept construction & 1.000 & 0.950 \\
Judgment on concept features & 1.000 & 0.902 \\
Design expression & 1.000 & 0.853 \\
Judgment on design expression & 1.000 & 0.655 \\
Value proposition & 1.000 & 0.829 \\
Assessment of value proposition & 1.000 & 0.824 \\
\hline
\end{tabular}

Table 5 The rotated component matrix

\begin{tabular}{llrr}
\hline Activity category & \multicolumn{2}{l}{ Component } \\
\cline { 2 - 4 } & 1 & \multicolumn{2}{l}{3} \\
\hline Value proposition & 0.906 & 0.041 & 0.045 \\
Assessment of value proposition & 0.893 & 0.133 & 0.119 \\
Design expression & 0.831 & 0.400 & -0.049 \\
Judgment on design expression & 0.706 & 0.382 & 0.107 \\
Concept construction & 0.130 & 0.966 & -0.009 \\
Judgment on concept features & 0.380 & 0.865 & 0.101 \\
Problem definition & 0.022 & -0.052 & 0.851 \\
Judgment on problem definition & 0.090 & 0.112 & 0.780 \\
\hline
\end{tabular}

\section{Strategies for concept generation}

Through the data analysis on the frequency of designer's activities (see Table 3), we extracted three common factors, F1, F2 and F3, which explained $37.20 \%, 25.27 \%$ and $17.17 \%$, respectively, and accounted for $79.63 \%$ of the total variance. This indicated a good effect of the common factors on explaining the original variables (Table 4). In the next step, a factor analysis model was established through varimax rotation of the component matrix.

F1 includes four original variables: design expression, judgment on design expression, value proposition and assessment of value proposition. F2 is mainly decided by concept construction and judgment on concept features, while F3 is decided by the remaining two variables: problem definition and judgment on the definition (Table 5). More details of the common factor score of the 21 teams can be seen in Table 6. Taking the scores of three factors as variables, a hierarchical cluster analysis was performed to extract the characteristics of concept generation strategies. The analysis was done by using the Ward method and squared Euclidean distance method in SPSS 19.0. We compared the results of different numbers of cluster, and as a result, we identified six types of concept generation strategies (see Table 6).

Type 1 is a strategy emphasizing design expression. This strategy focuses on the discussion on what the concept is for and how it is implemented. Except for T1, it is observed that the scores on F2 for T9, T10 and T21 are lower than zero. Only T21 has a 
Table 6 Factor scores and results of cluster analysis

\begin{tabular}{lrrrrl}
\hline Team no & \multicolumn{1}{l}{ Factor 1 } & \multicolumn{1}{l}{ Factor 2 } & Factor 3 & \multicolumn{1}{l}{ F } & Type \\
\hline 1 & 0.64795 & 0.37235 & 0.85408 & 0.60 & 1 \\
2 & 0.47413 & -1.48893 & 0.33038 & -0.18 & 6 \\
3 & -0.36763 & 0.51022 & 1.98965 & 0.42 & 2 \\
4 & 0.18700 & -1.41384 & 0.76526 & -0.20 & 6 \\
5 & -0.72819 & 0.71829 & -0.99381 & -0.33 & 3 \\
6 & 2.84084 & 1.31613 & 0.04244 & 1.75 & 5 \\
7 & -0.51591 & 0.65644 & -0.45800 & -0.13 & 3 \\
8 & -0.62715 & 0.48113 & -0.55763 & -0.26 & 3 \\
9 & 0.65201 & -0.20358 & 0.06111 & 0.25 & 1 \\
10 & 0.75909 & -0.01645 & 0.05826 & 0.36 & 1 \\
11 & -0.52560 & -0.79193 & -0.71246 & -0.65 & 6 \\
12 & -0.85045 & -0.53058 & -1.65096 & -0.92 & 6 \\
13 & 0.04112 & 0.81143 & -0.60039 & 0.15 & 3 \\
14 & -0.43705 & -1.32331 & -0.30590 & -0.69 & 6 \\
15 & -0.42016 & -1.26810 & 0.17675 & -0.56 & 6 \\
16 & -1.08425 & -0.23327 & 0.22009 & -0.53 & 6 \\
17 & -0.52454 & 1.55859 & 2.09153 & 0.70 & 2 \\
18 & -0.84484 & 1.06830 & -0.50786 & -0.17 & 3 \\
19 & -0.68135 & 1.49709 & -0.94441 & -0.05 & 3 \\
20 & -0.20508 & -1.14398 & 1.44903 & -0.15 & 4 \\
21 & 2.21003 & -0.57600 & -1.30719 & 0.57 & 1 \\
\hline & & & & &
\end{tabular}

score on F3 lower than - 1. These are indications of a relatively low frequency of concept construction activities. For these teams, it could be more important to search better ways to complete design work for the well-defined concepts based on a full analysis of problem scenarios than to spend much time extending or evaluating the concept points.

T3 and T17 feature scores lower than zero on F1, so they were grouped into the second type (i.e., Type 2) of concept generation strategy. This type demonstrated high activity of team members in the divergent step for both problem definition and concept construction. Students raised a considerable number of design issues in the definition stage and accordingly developed the concept, which is mapped to the problem. They took measures such as the retrieval of personal experience and contextual description to consider whether the concepts were reasonable to cope with social issues.

Six teams fall into a category called Type 3, with a factor score on F1 lower than or near zero. The F3 scores for all the teams were lower than zero. Obviously, Type 3 is a concept-oriented strategy. Students developed so many concepts with a large part of their time in an attempt to balance the interests of all stakeholders. Driven by a desire to attend to every aspect of the problem, this strategy was adopted by students to integrate the elements of the concept into a comprehensive proposal to form a service system. This strategy can contribute to enriching the design concept, with multiple alternatives having been provided. However, in most cases, many of the concept features were discarded in a subsequent convergent step. Therefore, only a few design concepts were further developed and expressed with sketches. 
The strategy of T20 was typical of Type 4, which focuses on gathering information from both external sources and personal knowledge to better define the problems. T20 took a long time to explore variations in the design concept for its work, which is why its scores on F1 and F2 were lower than zero. There seemed to be a systematic plan for the two students to guide their problem definition. According to our observation, they showed two directions to gather information: one was to have insight into the social context, and the other sought to build a problem scenario through user study tools. They selected valuable information only in a decisive manner, and as such, fewer convergent activities were recorded.

T6 allocated time quite evenly to different activities to express ideas as often as possible, with the two students interacting with each other more frequently than all the other teams in both divergent and convergent activities. We named this strategy Type 5, which is characterized by the balanced and full use of all the design activities. The remaining seven teams fell into Type 6, featuring a very low score on the three common factors. For F2, all the teams scored lower than zero, while five teams scored lower than zero for F1 and three for F3. These data implied that the participants did not perform enough activities for ideation and thus produced a limited number of concepts.

As suggested by the statistical results, there were significant differences in terms of frequency between divergent and convergent activit***ies (the Wilcoxon signed-rank test: problem definition: $\mathrm{Z}=-2.44, p<0.05$; concept construction: $\mathrm{Z}=-4.02, \mathrm{p}<0.001$; design expression: $\mathrm{Z}=-4.02, \mathrm{p}<0.001$; value proposition: $\mathrm{Z}=-3.01, \mathrm{P}<0.01$ ). Pairwise comparisons revealed significant differences between divergent activities. The frequency of concept construction was significantly higher than that of problem definition $(Z=-2.79$, $\mathrm{p}<0.01)$, design expression $(\mathrm{Z}=-2.42, \mathrm{p}<0.05)$ and value proposition $(\mathrm{Z}=-4.02$, $\mathrm{p}<0.001)$.

\section{Concept generation modes}

A journey map with the timeline can illustrate the occurrence of design activities as well as the divergence-convergence process. By extracting the similarities in the structure of journey maps, we summarized eight concept generation modes. Figure 5 provides the journey maps of eight teams, each representing one of these modes. These eight journey maps will be clarified in a subsequent part of this paper as examples to show the characteristics of concept generation modes. An overview of these characteristics is shown in Fig. 6 in the form of diagrams.

\section{Mode 1: Filtering}

T1 was the only team working with Mode 1, a choice-based mode by which one or more of the design initiatives could be picked out from a network of undefined problems and design concepts. The initiative(s) are further developed into a comprehensive solution. To better describe this mode, we compared it to the process in which sand is filtered and gradually accumulates (Figs. 5a, 6a). Before selecting the problems of future medical care, T1 enumerated a number of design objectives, among which the relief of anxieties was prioritized. However, this design objective and others, such as a redesign of online registration and health QR codes, were removed considering some restrictions. Even though many problems were described in the early stage with concepts having been constructed, students 


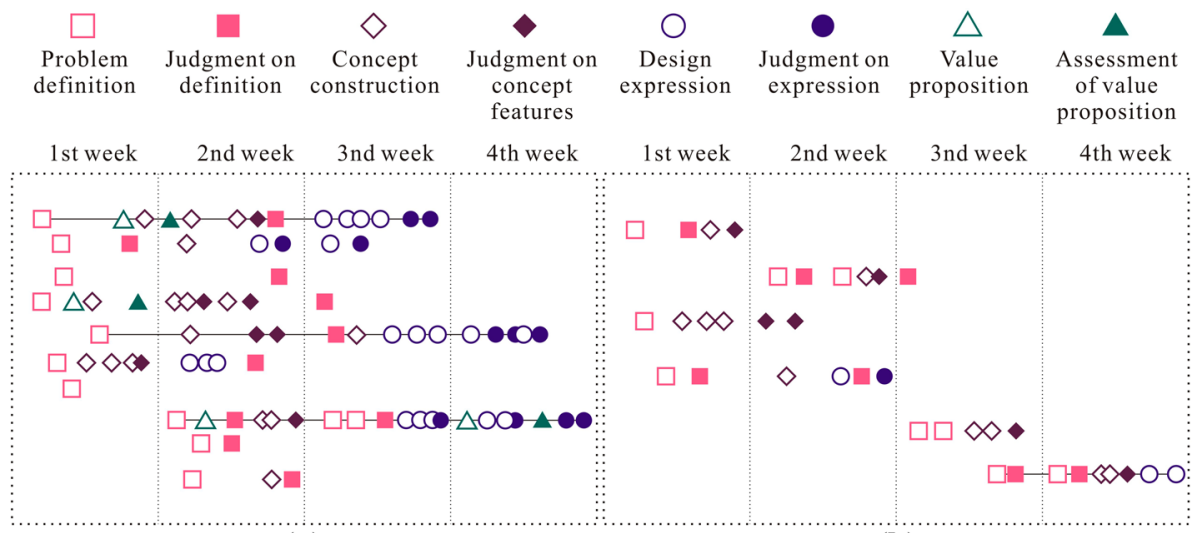

(a)

(b)

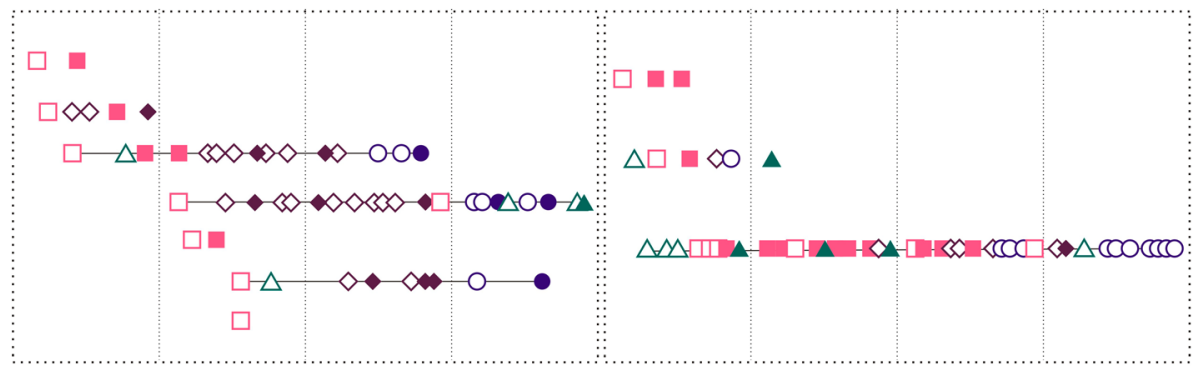

(c)

(d)

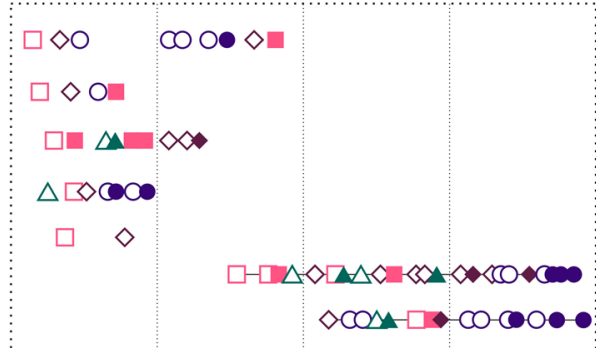

(e)

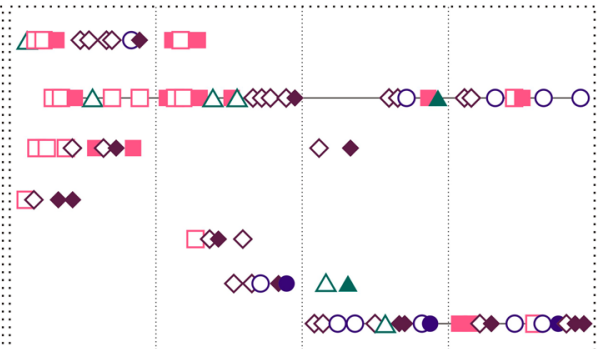

(f)

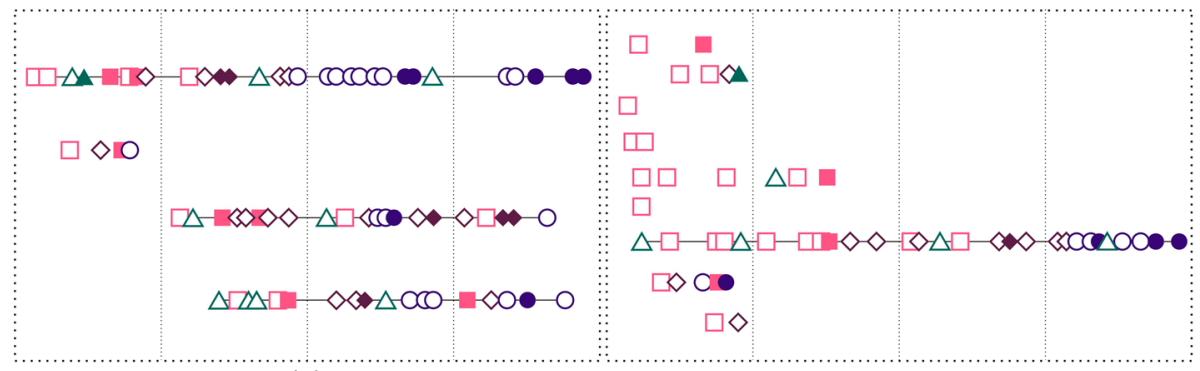

(g)

(h)

Fig. 5 Journey maps of the design process of representative teams for each concept generation mode: T1 for Mode 1 (a), T16 for Mode 2 (b), T7 for Mode 3 (c), T4 for Mode 4 (d), T9 for Mode 5 (e), T6 for Mode 6 (f), T10 for Mode 7 (g) and T20 for Mode 8 (h) 


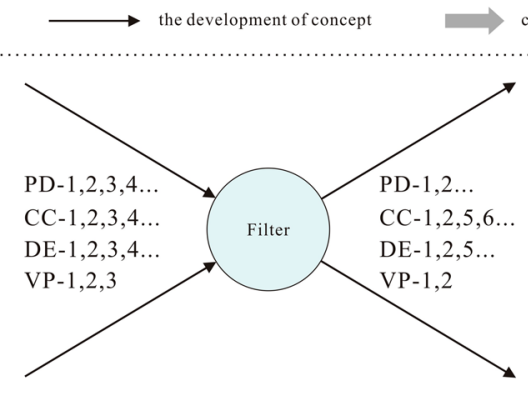

(a)

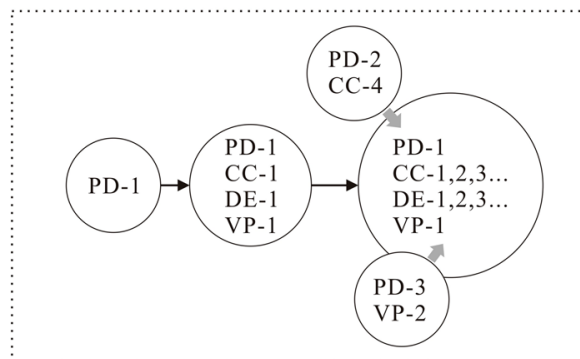

(c)

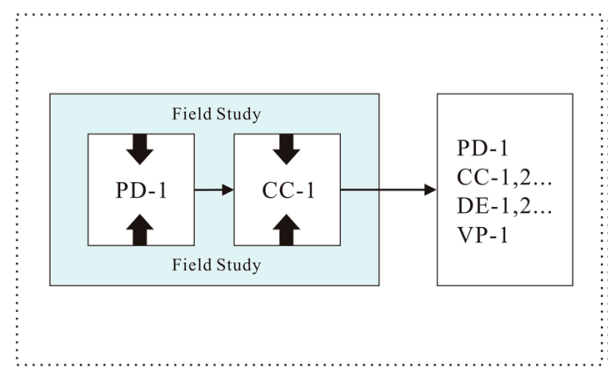

(e)

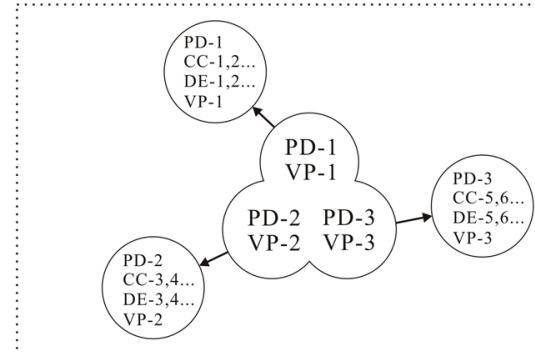

(g) combination $\longrightarrow$ extracting information

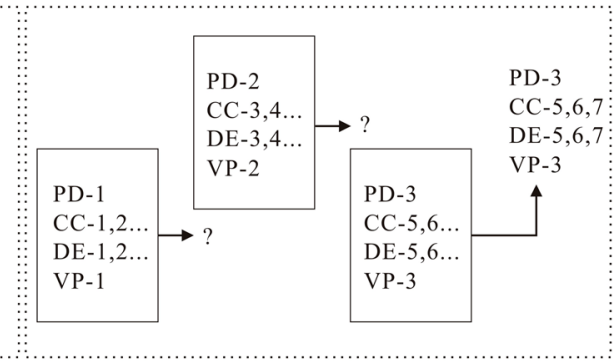

(b)

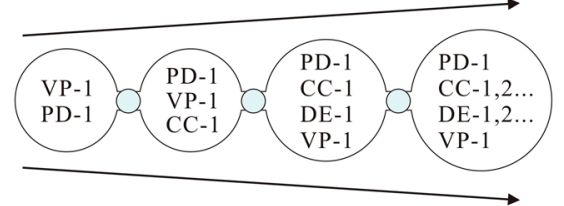

(d)

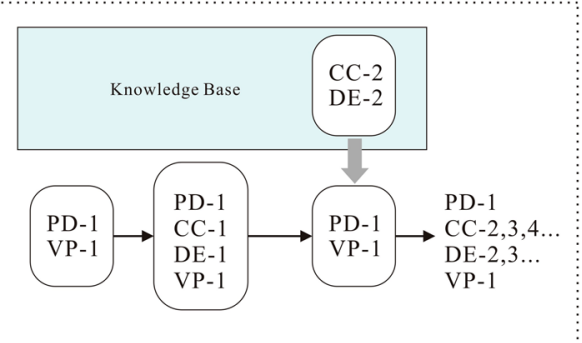

(f)

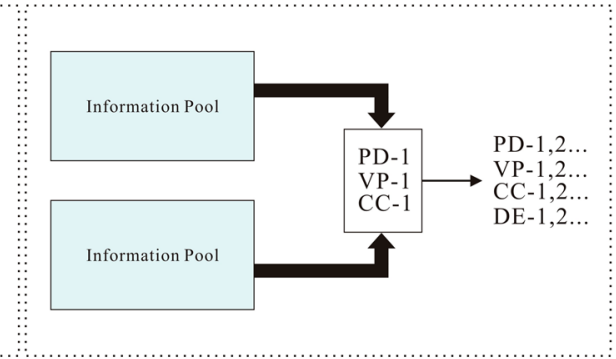

(h)

Fig. 6 The diagrammatic form of eight concept generation modes: Mode 1 (a), Mode 2 (b), Mode 3 (c), Mode 4 (d), Mode 5 (e), Mode 6 (f), Mode 7 (g) and Mode 8 (h) 
selected only three main concepts: a medical forum, a sign language service for clinics, and a self-reporting system of illness for deaf mutes.

P1: "We found that many of the design objectives we set in the very early phase are somewhat too complex to be handled by designers or the service platforms alone. I mean, these are subjects describing a sort of ultimate aim, for example, relieving anxiety of patients, facilitating communication between individual patients or between patients and doctors. And besides, some of our design concepts for such objectives seemed to resemble extant design cases. More importantly, some problem scenarios, in our view, emerge, as there exist a number of structural contradictions."

P2: "Yes, they need a comprehensive solution with changes in infrastructure, system or even human nature."

P1: "We had no way but to look for new problem scenarios to make a difference, and we finally focused on deaf mutes because we suspected that vulnerable groups could be a good subject for proposing creative design concepts."

P2: "In my experience, the transfer of the target group to disabled people was proven to be promising in generating more workable product design concepts. Through a field study, it was found that services for deaf mutes were lacking in both urban and rural hospitals in our country. It is difficult for them to describe the illness and symptoms to a doctor. Hence, we saw the opportunity for a self-report system in which more efficient sign language service is offered. This system can also be combined with the online treatment platform to provide patients with better remote diagnoses."

\section{Mode 2: Superseding}

Mode 2 features the repetition of proposing design concepts based on an ill-defined problem (Fig. 5b, 6b). Following this mode, seven teams' work failed to abstract the core of conflicts from social phenomena as a result. Some teams even skipped the judgment of problem definition as often as they did for concept design. Taking T16 as an example, the two students came up with ideas such as a portable disinfectant, finger disinfection and bacteria detector to prevent home care workers from being discriminated against in the postepidemic era. These ideas were presented one after another but with no elaboration on the reason why there is always mutual distrust between care workers and employers' families. Here is a dialog on developing the idea of a portable disinfectant:

P32: "You mentioned that the disinfection bag can transmit data to the employer's phone in order to know where the care worker has been, however, this is definitely a kind of surveillance and no one likes it."

P31: "Well, or just a bag or a package with the UV disinfector inserted inside...".

P32: "A new question. I'm not sure if there is a need to separate the worker's personal items from food. I need to check...".

P31: "Wait a minute. Uh. What if, in this case, the bag's surface was contaminated with virus. What can we use to disinfect it when the care worker comes back home?".

As observed in the behaviors of T16, students working with Mode 2 were not used to analyzing a problem immediately after it was presented; they would rather temporarily consent to its necessity and begin to develop ideas. They rarely reflected on the repeated failures in proposing solutions and were not used to comparing multiple concepts they had already developed. The final concept of T16 was a web platform for equal dialog that enables employers and care workers to post their secrets and complaints anonymously, allowing for the exchange of opinions between the two groups. 


\section{Mode 3: Attaching}

When multiple problems have been defined, designers will normally specify one among them and generate concepts for it. In Mode 3, a problem of this kind is supported by the related problems, as if small particles are absorbed and stuck onto the surface of a larger substance (Fig. 6c). Figure 5c depicts the thinking process of T7, a team concentrating on the promotion of children's awareness of anti-epidemic measures. This design objective requires the participation of different stakeholders, including parents, teachers and the community, in children's education. Therefore, two additional design ideas were attached to the main concept called "children's daily attendance of disinfection". One is a role-playing game within the family unit, and the other involves changing the child's role into the persuader for preaching anti-epidemic knowledge to the community under teachers' guidance. As seen in Fig. 5c, these ideas were developed from independent issues, i.e., how to disinfect, educate and share knowledge, and were integrated just because a particular group of people could participate in all the activities. With little convergent thinking for the evaluation of the primary concepts, the final concept constructed by $\mathrm{T} 7$ retained a number of subsidiary ideas that were attached to the main one, as there is a connection or some similarity between them.

\section{Mode 4: Node}

As the only representative of Mode 4, T4 persisted with the original idea derived from a fast divergence-convergence in combination with a distinct value proposition during the whole design process. At the beginning of the concept generation, the two students pointed out that the sense of guilt we all have on a high-calorie diet is a psychological burden; they wanted to release the burden by doing something to "atone for the sins". Through an in-depth interview, this design objective was confirmed, laying a foundation for the following concept generation. Students then defined the varying engagement level of a somatic game (Fig. 7a) to compensate for the sense of guilt. After that, they detailed the interaction design of the game interface and the design of the game device. By and large, a succession of "nodes" (Fig. 6d) existed in the design process of T4 as an indication of the fact that T4 took a further step only when the tasks in the previous design phase were complete. These tasks are generating an initial idea, developing the primary concepts by elaborating a problem, defining the social value of its potential solution, and constructing the whole concept.

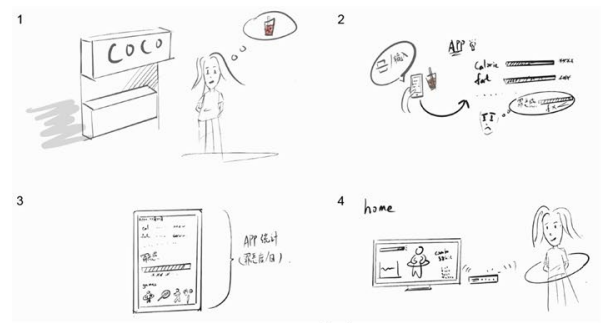

(a)
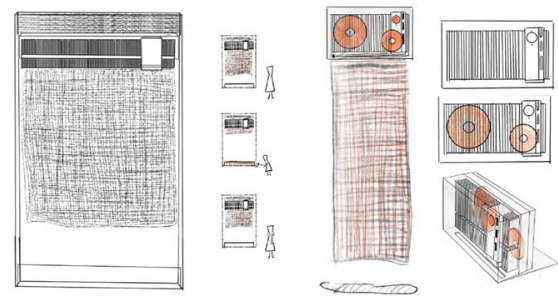

(b)

Fig. 7 The design concept of T4 (a) and T6 (b) 


\section{Mode 5: Mutation}

"Mutation" is the metaphor of Mode 5, as it occurs when designers have gained unexpected information that is useful for generating preliminary but creative concepts from the field study. In some cases, such information directly helps problem definition and concept construction (Fig. 6e). In social design practices, this mode is far from rare and mostly emerges when an interview or codesigning shows designers the opportunity for resource exchange or the connection between interests. For example, T9 discovered by chance that some of the interviewees were gardening enthusiasts. Inspired by this finding, the team designed a flowerpot equipped with a breathing lamp. The lamp displays the fitness state of friends by personifying the flower with a light effect. By doing so, the interaction with friends could be strengthened. Team members briefed the researchers on their trains of thought in the self-reflection session.

P18: "The vision we defined is how to maintain the physical and mental health of people during the quarantine, but we had no idea about how to start the design. We then interviewed a couple of our intimates to know what they like and what they would do at home. With three of the interviewees having been growing flowers, we decided to try the effect of mapping the fitness diary to the growth of flowers. My partner pointed out that people would not accept the withering of flowers; so, I generated a new idea about the indicator of growth, that is, using the light effect of different colors and brightness."

\section{Mode 6: Borrowing}

Industrial designers are sensitive to the market and frequently conduct competitive analysis. They also are used to methods such as analogical reasoning or conflation to create new concepts. These methods are effective instruments for social design when there is a need for borrowing concept features from the knowledge base of the designer (Fig. 6f) to enhance the creativity of solutions. This "borrowing" mode was represented by T6 (Fig. 5f) and T17. After two weeks debating how to present the information of people who died of the pandemic for memories of them, T6 made little progress toward a satisfactory design proposal. The two students searched the internet for new information and were inspired by the idea of objectifying increasing data. With this idea, they devised a machine that produced fabrics for the public to get it free. The length of the fabrics depends on the activeness of the social media accounts of the departed (Fig. 7b). This was designed to be a medium for keeping the collective memory and to promote a sense of community. The "borrowing" mode can usually be observed when designers need new approaches to the design objective, which has been confirmed with well-defined problems.

\section{Mode 7: Dividing}

T10 was interested in the "invasion" of suburban wild animals when the city was on lockdown. Team members intended to introduce the dilemma of wild animals' survival in metropolises with a well-designed educational game. They studied the needs of related stakeholders and became aware of the importance of scientific data for the game and the necessity of evaluating its educational benefits. 
P19: "That is what I persist in. Regardless, the game tasks should be triggered when we detected some happenings in a raccoon dog's life. These occurrences are indications of changes in survival. I do not see any differences if we make a game in which the tasks are fixed and preset."

P20: "I understand it. As you said, the location and physiological data of raccoon dogs collected by scientists should be synchronized with the game process; thus, the players can clearly know how the inhabitants will interact with these small animals."

P19: "Correct. Such a design enables us to immerse in animals' lives, providing a new perspective on the contradiction between men and nature. Then, scientists explain the game tasks and the observed data to game players. This is a learning process in addition to a game experience."

The two students clarified the close relationship between their design goals and defined a sequence of public activities for introducing the suburban ecosystem (Fig. 5g). The students presented three concepts: GPS detection of the locations of raccoon dogs, a locationbased digital game simulating these animals' actions for survival, and children-oriented popularization lectures after game-playing. These constituted the three main aspects of the "training for young citizen scientists" project (Fig. 8). This mode can, in our study, be compared to the process of cell division (Fig. 6g). Designers usually plan a multistep activity first. Since the activity can be divided into several subactivities that are networked, and each involves multiple stakeholders, the final concept as a whole contains a great amount of detail on the subactivities, and its main theme remains unchanged. In addition to T10, T13 and T21 are the other two teams using this mode to generate concepts.

\section{Mode 8: Matching}

To determine the potential needs of the employment market in the postepidemic era, much work has been done for T20 to collect existing recruitment patterns by analyzing every case the students can find. In the meantime, the two students listed a variety of scenes requiring new jobs as the problem scenarios. During this process, an "information pool" was established (Fig. 6h). In the next step, the designers' efforts were centered on finding a good match between the background information and the problem scenario to complete

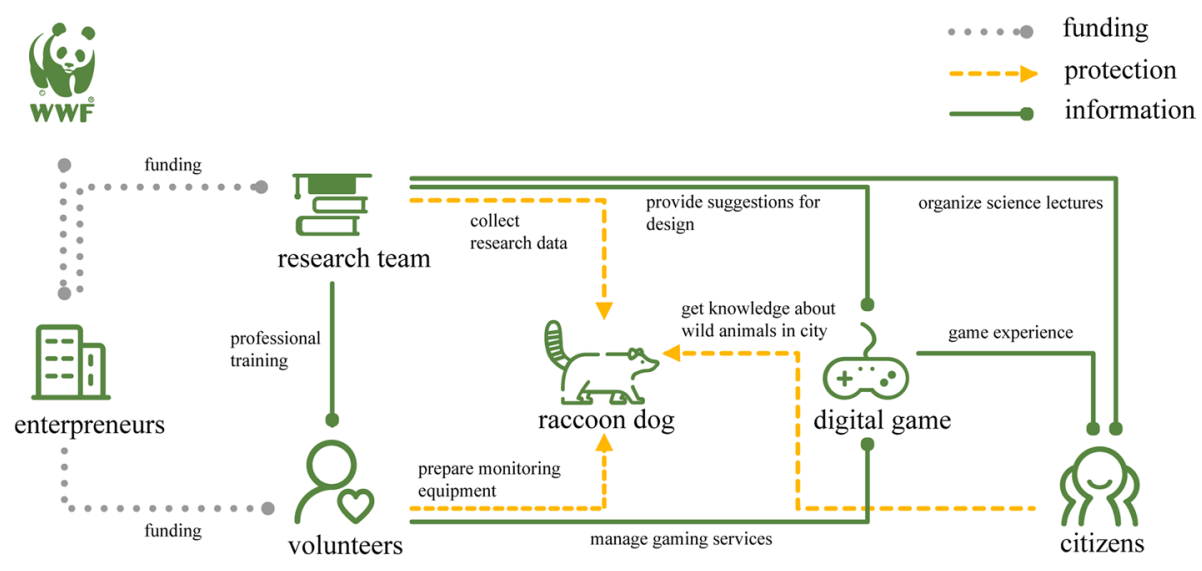

Fig. 8 System map in the design concept of T10 
problem definition and concept construction at the same time. Compared to the high frequency of divergent activity for establishing the information pool, team members evaluated the matching only by a few convergent activities (Fig. 5h). They considered project-based employment a good solution for the unemployment of low-skilled marginalized groups and selected the management of shared bikes as the problem scenario. The final design concept was described as motivating elderly people to assist in the balanced distribution of shared bikes by rewarding them with a random sum of money on the service provider platform.

\section{Assessment of the concepts}

Figure 9a shows the expert score of the originality of students' concepts for each strategy and mode. Regarding the strategy, Type 5 received the highest score in terms of originality, followed by Type 1, Type 4, Type 2, Type 3 and Type 6 . Significant differences in originality were found between these strategies by pairwise comparison (Type 5 vs. Type 2: $\mathrm{U}=54.50, \mathrm{Z}=-3.04, \mathrm{p}<0.01$; Type 2 vs. Type $6: \mathrm{U}=726.00, \mathrm{Z}=-2.11, \mathrm{p}<0.05$; Type 4 vs. Type 3 : $U=136.50, Z=-3.878, p<0.001)$. According to these statistics, the concepts

Strategy

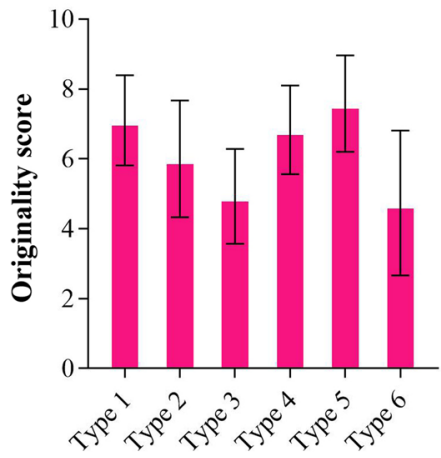

(a)

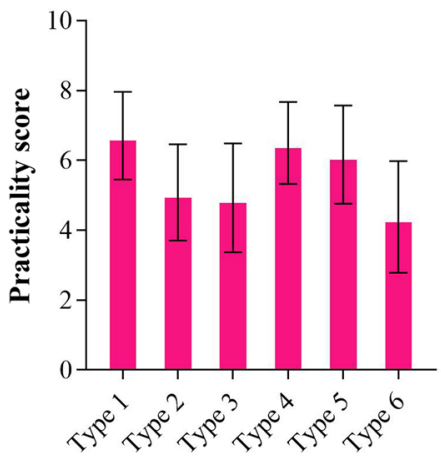

(c)

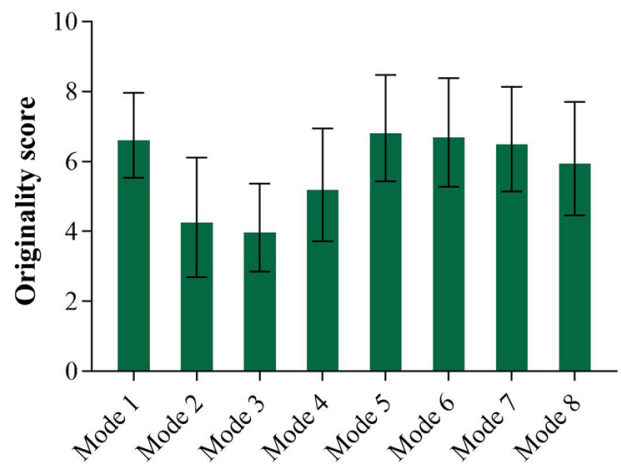

(b)

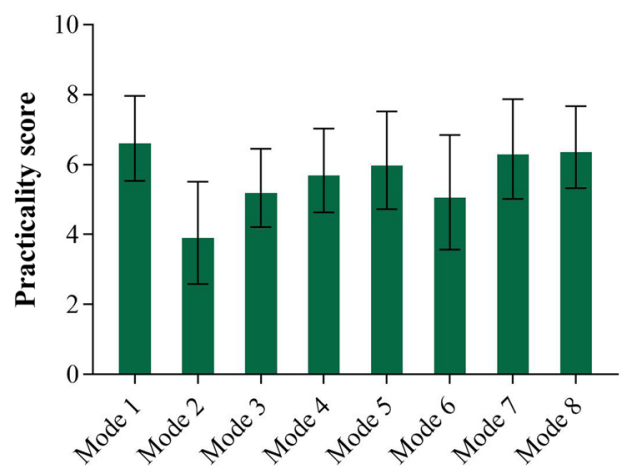

(d)

Fig. 9 Expert scores of all the students' concepts 
generated with Type 3 and Type 6 are significantly less creative than those generated with Type 5, Type 1 and Type 4.

Regarding the concept generation mode, the originality score of the concepts generated by Modes 2, 3 and 4 is much lower than those generated by the other modes (Kruskal-Wallis's H test: $\chi^{2}=83.53$, df $=7, p<0.001$ ), see Fig. 9b. Since the concepts of T1, T4 and T20 are the only projects that embody Mode 1, Mode 4 and Mode 8, respectively, the results may change with increasing team numbers. Future research should further focus on the contribution of the strategies and modes of high-quality social design concepts.

The practicality scores of the strategies and modes are detailed in Fig. 9c and 9d. Statistics showed that the scores of different strategies were significantly different (Kruskal-Wallis's H test: $\chi^{2}=70.75, \mathrm{df}=5, \mathrm{p}<0.001$ ). For Types 1,4 and 5 , the practicality scores were higher than those of Types 2, 3 and 6. Mode 1 received the highest expert score in practicality, followed by Modes 8, 7, 5, 4, 3, 6 and 2. The scores of different modes also were significantly different (Kruskal-Wallis's $H$ test: $\chi^{2}=96.54, \mathrm{df}=7, \mathrm{p}<0.001$ ). In the next part, we discussed the reasons for the differences in design quality of all strategies and modes.

\section{Discussions and conclusions}

\section{Characteristics of industrial designers' thinking}

This study conducted an experiment to identify the characteristics of ID students' thinking when they implement a social design project for the first time. It was found that students had some preferences in the selection of design themes, and their concept generation strategies and modes were diverse. ID students have the following characteristics in selecting a theme of social design.***

1. With a desire to improve human life, many ID students participated in our research and naturally regarded problem solving as the goal of social design. The students presented some concepts with a variety of purposes, but they viewed these purposes as the byproduct of or the means to problem solving.

2. Another worth-mentioning characteristic of industrial designers, the consciousness of serving target users, is shown in their discussions on values. Some of the values, such as convenience and emotional needs, suggest a sense of user-centered design that attaches more value to the feelings or experience of users than self-actualization, social relationships or community life. Identifying users' characteristics also is essential to recognize the opportunities for developing usable or attractive designs, and for this reason, most of the students conducted persona analysis. Such designs are allowed for mass production and play a critical role in many concepts presented by students. Only T6 and T21 mentioned "behavioral art" when introducing their concepts, as one member of T21 said in the self-statement session:

"We initially intended to redesign the thank-you letters sent with the take-out food, or rather... I mean, to guide the restaurant owners to draw pictures on such letters, making them a narrative...picture-story book. The pictures can be the window for branding, self-presentation or sharing owners' lives and emotions and thus impressing diners or arousing their interests. We basically want to deepen the social connection between nonresident entrepreneurs and community 
residents. This connection can become the first step into a network of food services..."

3. Without any external intervention, ID students are more likely to propose an interaction or a product design concept, or a product service system design, to be the solution of a social and societal issue. In our study, many of these design concepts are expressed in a tangible form and can be applied across scenarios. In contrast, the strategic design for social systems, policies, public services and community relationships is not an important element of students' concepts.

As can be observed in this study, teams varied in their frequency of design activities. The high frequency of certain activities indicates that these were what a team relied on more to conduct idea generation. For one-third of the participating students, there was no tendency that they performed specific design activities more frequently during their design process. It also should be noted that the value-driven ideation strategy was absent. This could be attributed to the inadequacy in learning sociological theories that may help in forming, evaluating or reflecting ideas through the analyses of social meaning. Moreover, in the factor analysis, design expression and value proposition were clustered into one main factor. This result is unexpected but reasonable, as we noticed that students preferred to propose the values in justification of a finished concept instead of using them to support the development of the concept. Furthermore, we observed divergent activities more frequently than convergent activities. This might be because the judgments on propositions were made by students without much discussion; that is, students might actually have made a decision on whether to keep or discard an idea, but there was no verbal expression for that.

The investigation of the journey of divergent and convergent thinking activities revealed eight concept generation modes. Modes 1 and 8 are two different approaches to compressing the problem space. In Mode 1, the selection of undefined problems depends on a comprehensive evaluation of several design initiatives that are already or roughly formed. In Mode 8, designers gather information in relation to the social background and problem scenario; then, they select the information that can be interrelated. Established on a well-defined problem, Mode 4 is a design progress where students can develop a concept deeply, with several convergent steps, to seek a systematic solution. Mode 7 is used to structure a design problem by providing a view of its components. These components may help in constructing a problem network in the preliminary stage of social design. Mode 5 features a working method of taking notice of potential opportunities for design in the firsthand research data to generate creative ideas. Mode 6 retrieves external information from the designer's reservoir of knowledge to merge it into idea generation. External information usually refers to similar problem scenarios and solutions by which designers can be inspired. In Mode 2, participating students repeat the pattern of trial-and-error until they come up with a satisfactory concept. In some cases, participants first established a core concept and then tried to connect the relevant concepts with it to form a system. This mode is described as Mode 3.

\section{Evaluating the concept generation strategies and modes}

Expert designers evaluated the design concepts of the 21 teams in terms of originality and practicality. Significant differences were found in these two dimensions across concept generation strategies. We observed many more divergent activities of T6 in all the stages of 
concept generation than the other teams, which characterized a distinctive strategy called Type 5. Theoretically, this strategy can result in better design concepts, but it also challenges novice designers. Compared with the other teams, T1, T9, T10, T20 and T21 put more effort into problem definition. This contributed to more divergent and convergent activities in the early stage of concept generation, so the quality of concept was greatly improved. There were 13 among 21 teams adopting these two strategies: Type 3 and Type 6 . The number of design activities encoded in the design process of these teams is smaller. Moreover, concept construction accounted for a large part of the encoded activities of these teams. Table 6 also shows that the frequency of concept construction was much higher than that of the other activities in our study. These results suggest that ID students, as novices in social design, tend to be more focused on developing the concept than the full analysis of the social issues. The effort students invested in constructing concepts did not make them more original and practical, perhaps because they did not know how to use effective methods to develop truly valuable concepts.

Different modes of concept generation also may have an effect on the quality of social design concepts, as we found that the concepts developed with Modes 1, 5, 6, 7 and 8 are more original, and those with Modes 1, 4, 5, 7 and 8 are more practical. On the whole, Modes 1, 5, 7 and 8 are journeys with a well-planned working flow, by which the designers can better interpret social issues and explore more potential opportunities. Therefore, these modes could be more effective in generating social design concepts with high quality.

One problem with Mode 4 is that the designers develop the initially constructed core concept so constantly that they will not take much action to extend this concept. In this way, the designers have to be fixated on a defined problem or social value on which the subsequent work is based. This may be an explanation for the lack of originality of T4's concept. The innovation of the concept developed with Mode 6 comes from the knowledge base of designers rather than field study or codesigning with stakeholders. This may lead to some interesting ideas but with less feasibility. We recommend that this concept generation mode can be more closely combined with the participating design in action.

In Mode 2, students defined a problem without much discussion and then made a concept in response to it. Once the concept was not supported by teammate or field study results, it was discarded, and everything went back to the beginning. This meets Ahmed et al. (2003) description of novice designers. The reason for developing concepts with Mode 3 may be the lack of effective judgments on problem definition before generating ideas. Students seemed not very conscious of the necessity of such judgments and thus could not identify the core problem even after the concepts were fully developed. In this case, they would combine all the concepts together instead of dividing a core problem into parts like Mode 7. The expert scores suggest that these two modes may have negative impacts on the quality of social design initiatives; however, there is a notable number $(n=11)$ of teams using these two modes.

\section{Implications for design education}

Industrial design students have advantages of domain expertise when they become involved in social design. First, ID students are familiar with the design flow, from defining problems and idea generation to field testing and iteration. These stages also are the key components of a toolkit for social design teaching presented by Yavuzcan et al. (2019). Second, the experience ID students have with user study can pave the way for them to conduct contextual research. They learn how to organize interviews and are trained to observe and 
Table 7 The open questionnaire used for self- statement

Self-statement form

Question 1

Question 2

Question 3

Question 4

Question 5

Question 6

Question 7

Question 8
What is the social problem or issue on which your concept is based?

Try to briefly describe the core of your concept

What target people are your concept is developed for?

To what extent and in what ways will your concept affect the society?

What makes you aware of the social issues that your concept is aimed at?

What are the difficulties that you encountered in the process of concept generation?

What are the shortcomings and limitations of your concept?

What materials and external information do you use as references to analyze problems and generate concepts?

empathize with end-users. Third, ID students should be creative and used to work in collaboration with different partners. These merits allow ID students to develop social design initiatives independently, even without design expertise in this domain.

As novices, ID students' thinking about social design is nevertheless limited and needs to be improved in the following aspects. First, a social design project goes with the meaning-making process. This is not an easy task for many ID students with little knowledge or understanding of the sociological, historical and cultural theories. Most of the students, at least in our study, saw design as a response to the problematic situation rather than as a tool to perceive or participate in social changes. Second, participating students' designs began with problem analysis but usually ended with insufficient problem formulation. We observed some characteristics of the students' concept generation strategies and modes that would limit them to reframe a problem domain in which a small effect could be made with designer involvement to energize the community. Third, students took certain activities more frequently. Some teams paid more attention to the analysis of human needs, social phenomena and their contributing factors in the early stage of concept generation. Some teams allocated much time to the social value of design, while some intended to optimize the design proposal with much effort in concept construction. Only one team, T6, tried to balance every activity to develop the concept. Fourth, more divergent activities were revealed in the case study than convergent ones, which included reflections, evaluations and debates. These findings raise important issues for future teaching practice in relation to nurturing social designers.

In the whole study, we did not attempt to examine the effects of the coworking of designers with a specific group of citizens, public partners or private partners on idea generation. There also are differences in idea generation that might exist between a single designer and teamwork, as well as between industrial designers and the others. Further study is needed to explore these questions that have not yet been answered.

\section{Appendix}

See Table 7.

Acknowledgements The authors would like to thank all participants in this research. 


\section{Declarations}

Conflict of interest The authors declare that they have no conflict of interest.

\section{References}

Ahmed, S., Wallace, K. M., \& Blessing, L. T. M. (2003). Understanding the differences between how novice and experienced designers approach design tasks. Research in Engineering Design, 14(1), 1-11.

Akdur, S. E., \& Kaygan, H. (2019). Social design in Turkey through a survey of design media: Projects, objectives, participation approaches. The Design Journal, 22(1), 51-71. https://doi.org/10.1080/14606925. 2018.1560592

Armstrong, L., J. Bailey, G. Julier., \& L. Kimbell. (2014). Social design futures: HEI research and the AHRC. Brighton: University of Brighton; London: Victoria and Albert Museum.

Atman, C. (1999). A comparison of freshman and senior engineering design processes. Design Studies, 20(2), $131-152$.

Atman, C., \& Bursic, K. M. (1998). Verbal protocol analysis as a method to document engineering student design processes. Journal of Engineering Education, 87(2), 121-132.

Atman, C., Cardella, M. E., Turns, J., et al. (2005). Comparing freshman and senior engineering design processes: An in-depth follow-up study. Design Studies, 26(4), 325-357.

Banathy, B. H. (1996). Designing social systems in a changing world. Plenum Press.

Bobbe, T., Wölfel, C., Krzywinski, J. (2017). Participatory research with refugees - can industrial design engineers implicitly cope with social design problems? The 19th International Conference on Engineering and Product Design Education, Oslo, Norway.

Bryson, M. (1991). Going beyond the problem as given: Problem solving in expert and novice writers. Complex Problem Solving: Principles and Mechanisms, 61-84.

Carney, G. M., Dundon, T., \& Léime, A. N. (2012). Participatory action research with and within community activist groups: Capturing the collective experience of Ireland's community and voluntary pillar in social partnership. Action Research, 10(3), 313-330.

Chen, D., Cheng, L., Hummels, C., et al. (2016). Social design: An introduction. International Journal of Design, 10(1), 1-5.

Cross, N. (2008). Engineering design methods-Strategies for product design (4th ed.). Chichester.

Cross, N. (2010). Designerly ways of knowing. Springer.

Del Gaudio, C., Franzato, C., \& de Oliveira, A. J. (2016). Sharing design agency with local partners in participatory design. International Journal of Design, 10(1), 53-64.

DiSalvo, C., Lodato, T., Fries, L., et al. (2011). The collective articulation of issues as design practice. CoDesign, 7(3-4), 185-197.

Dorst, K. (1997). Describing design: A comparison of paradigms. PhD thesis, Netherlands: Delft University, 67.

Dorst, K., \& Valkenburg, R. (1998). The reflective practice of design teams. Design Studies, 19(3), 249-271.

Dwarakanath, S., \& Wallace, K. M. (1995). Decision-making in engineering design: Observations from design experiments. Journal of Engineering Design, 6(3), 191-206.

Easterday, M. W., Gerber, E. M., \& Lewis, D. G. R. (2018). Social innovation networks: A new approach to social design education and impact. Design Issues, 34(7), 64-76.

Finke, R. A., Ward, T. B., \& Smith, S. M. (1992). Creative cognition: Theory, research, and applications. MIT Press.

Hu, Y., Y. Guo and R. He. (2015). Mutual trigger effects in team-based ideation. In Proceedings of the 3rd International Conference for Design Education Researchers. 214.

Jégou, F., and E. Manzini. (2008). Collaborative services: Social innovation and design for sustainability. Polidesign. Milano.

Kang, L. (2016). Social design as a creative device in developing countries: The case of a handcraft pottery community in Cambodia. International Journal of Design, 10(1), 65-74.

Kiernan, L., Ledwith, A., \& Lynch, R. (2020). Comparing the dialogue of experts and novices in interdisciplinary teams to inform design education. International Journal of Technology and Design Education, 30, 187-206.

Kim, E., \& Kim, K. (2015). Cognitive styles in design problem solving: Insights from network-based cognitive maps. Design Studies, 40, 1-38.

Koskinen, I., \& Hush, G. (2016). Utopian, molecular and sociological social design. International Journal of Design, 10(1), 65-71.

Kruger, C., \& Cross, N. (2006). Solution driven versus problem driven design: Strategies and outcomes. Design Studies, 27(5), 527-548. 
de Lange, C., T. Wiegers and J. Vergeest. (2014). Social cohesion design, a course for designing community integrated product systems. In Proceeding of the International Conference on Engineering and Product Design Education, The Netherlands.

Levina, N., \& Ross, J. W. (2003). From the vendor's perspective: Exploring the value proposition in information technology outsourcing. MIS Quarterly, 27(3), 331-364.

Liu, Y., \& Chakrabarti, A. (2003). Towards an "ideal" approach for concept generation. Design Studies, 24(4), 341-355.

Luo, S., \& Dong, Y. (2017). Role of cultural inspiration with different types in cultural product design activities. International Journal of Technology and Design Education, 27, 499-515.

Manzini, E. (2015). Design, when everybody designs: An introduction to design for social innovation. MIT Press.

Margolin, V. (2002). The politics of the artificial. University of Chicago Press.

Markussen, T. (2017). Disentangling "the social" in social design's engagement with the public realm. CoDesign, 13(3), 160-174. https://doi.org/10.1080/15710882.2017.1355001

Melles, G and Z. Howard. (2012). Empower me-social design innovation for homeless families: Collective design creativity. In Proceedings of the 2nd International Conference on Design Creativity (ICDC2012), Glasgow, UK.

Melles, G., de Vere, I., \& Music, V. (2011). Socially responsible design: Thinking beyond the triple bottom line to socially responsive and sustainable product design. CoDesign, 7(3-4), 143-154.

Miettinen, S and M. Sarantou. (2017). Social design for services framework: Capturing service design for development framework. Design Management Association.

Mozdzynski, P. (2014). On some narrations in the (post)industrial design field. Zalacznik Kulturoznawczy, 1, 2014.

Papanek, V. (1985). Design for the real world: Human ecology and social change. Academy Chicago Publishers.

Philips, R., Brown, M., \& Baurley, S. (2016). Social responses to nature: Citizen empowerment through design. Journal of Design, 2(2), 197-215. https://doi.org/10.1386/dbs.2.2.197_1

Pugh, S. (1991). Total design: Integrated methods for successful product engineering (pp. 1-278). Addison-Wesley.

Roy, B., Castiglioni, A., Kraemer, R. R., et al. (2012). Using cognitive mapping to define key domains for successful attending rounds. Journal of General Internal Medicine, 27(11), 1492-1498.

Schön, D. (1983). The reflective practitioner: How professionals think in action. Basic books.

Shah, J. J., Kulkarni, S. V., \& Vargas-Hernandez, N. (2000). Evaluation of idea generation methods for conceptual design: Effectiveness metrics and design of experiments. Journal of Mechanical Design, 122(4), 377-384. https://doi.org/10.1115/1.1315592

Szentpéteri, M. (2009). Socially responsible design initiatives in Hungarian design education. ELISAVA Escola Superior de Disseny.

Thorpe, A., \& Gamman, L. (2011). Design with society: Why socially responsive design is good enough. CoDesign, 7(3-4), 217-230.

Tromp, N. (2013). Social design: How products and services can help us act in ways that benefit society. PhD Thesis, Technische Universiteit Delft.

Tromp, N., Hekkert, P., \& Verbeek, P. (2011). Design for socially responsible behavior: A classification of influence based on intended user experience. Design Issues, 27(3), 3-19. https://doi.org/10.1162/DESI_a_ 00087

van Leeuwen, J. P., Rijken, D., Bloothoofd, I., et al. (2020). Finding new perspectives through theme investigation. The Design Journal, 23(3), 441-461. https://doi.org/10.1080/14606925.2020.1744258

Wang, W., Bryan-Kinns, N., \& Ji, T. (2016). Using community engagement to drive co-creation in rural China. International Journal of Design, 10(1), 37-52.

Whiteley, N. (1994). Design for society. Reaktion Books.

Yang, C., \& Sung, T. (2016). Service design for social innovation through participatory. International Journal of Design, 10(1), 21-36.

Yavuzcan, H. G., Şahin, D., Gür, B., et al. (2019). An instructional model for social design education: A design project for stray animals including production-based learning approach. International Journal of Technology and Design Education, 24(1), 33-64.

Yilmaz, S., \& Seifert, C. M. (2011). Creativity through design heuristics: A case study of expert product design. Design Studies, 32(4), 384-415.

Publisher's Note Springer Nature remains neutral with regard to jurisdictional claims in published maps and institutional affiliations. 